\title{
LivDet 2013 Fingerprint Liveness Detection Competition 2013
}

\author{
Luca Ghiani ${ }^{1}$, David Yambay ${ }^{2}$, Valerio Mura ${ }^{1}$, Simona Tocco ${ }^{1}$, Gian Luca Marcialis ${ }^{1}$, \\ Fabio Roli ${ }^{1}$, Stephanie Schuckers ${ }^{2}$ \\ ${ }^{1}$ University of Cagliari - Department of Electrical and Electronic Engineering Italy \\ \{luca.ghiani, marcialis, roli\}@diee.unica.it, \{valeriomura, tocco.simona@\}gmail.com \\ ${ }^{2}$ Clarkson University - Department of Electrical and Computer Engineering USA \\ \{yambayda, sschucke\}@clarkson.edu
}

\begin{abstract}
A spoof or fake is a counterfeit biometric that is used in an attempt to circumvent a biometric sensor. Liveness detection distinguishes between live and fake biometric traits. Liveness detection is based on the principle that additional information can be garnered above and beyond the data procured by a standard verification system, and this additional data can be used to verify if a biometric measure is authentic.

The Fingerprint Liveness Detection Competition (LivDet) goal is to compare both software-based (Part 1) and hardware-based (Part 2) fingerprint liveness detection methodologies and is open to all academic and industrial institutions. Submissions for the third edition were much more than in the previous editions of LivDet demonstrating a growing interest in the area. We had nine participants (with eleven algorithms) for Part 1 and two submissions for Part 2.
\end{abstract}

\section{Introduction}

Among biometric systems, fingerprints systems are probably the best-known and widespread because of the fingerprint properties: universality, durability and individuality. Unfortunately it has been shown that fingerprint scanners are vulnerable to spoof attacks, i.e. it is possible to deceive a fingerprint system with an artificial replica of a fingertip. Therefore, it is important to develop countermeasures to those attacks.

Liveness detection, with either hardware-based or software-based systems, is used to check if a presented fingerprint originates from a live person or an artificial finger. Usually the result of this analysis is a score used to classify images as either live or fake.

To detect liveness, hardware-based systems employ a combination of additional sensors and software and may in- clude measurements outside of the fingerprint image itself while the software-based only use image processing algorithms to gather information directly from the collected fingerprint.

Since 2009, in order to assess the main achievements of the state of the art in fingerprint liveness detection, the Department of Electrical and Electronic Engineering of the University of Cagliari, and the Department of Electrical and Computer Engineering of the Clarkson University, have organized the Fingerprint Liveness Detection Competition.

The First International Fingerprint Liveness Detection Competition LivDet 2009 [1], provided an initial assessment of software systems based on the fingerprint image only. The second and third Liveness Detection Competition (LivDet 2011 [2] and 2013) were created in order to ascertain the current state of the art in liveness detection, including integrated system testing. LivDet 2011 and 2013 were both open to all academic and industrial institutions and contained two parts: evaluation of software-based systems in Part 1: Algorithms, and evaluation of integrated systems in Part 2: Systems.

In this paper, we describe the LivDet 2013 competition characteristics and we summarize the results achieved from the participants. Section 2 describes some of the fingerprint spoofing techniques and the liveness detection countermeasures. In section 3 the evaluation protocols of the algorithms and the systems are examined in depth. Section 4 presents the competition results and section 5 concludes the paper.

\section{Fingerprint spoofs creation}

There are two methods to create an artificial fingertip, the cooperative method and the non-cooperative method. In the cooperative method the subject pushes the finger into a plasticine-like material creating a negative impression of the fingerprint as a mold.

The mold is then filled with a material, such as gelatin, PlayDoh or silicone that will reproduce the fingerprint char- 
acteristics. In the non-cooperative method a latent fingerprint left on a surface is enhanced, digitized through the use of a photograph, and, finally, the negative image is printed on a transparency sheet. This printed image can then be made into a mold, for example, by etching the image onto a printed circuit board which can be used to create the spoof cast.

\section{Experimental Protocol and Evaluation}

The competition features two distinct parts; Part 1: Algorithms and Part 2: Systems, with separate protocols designed for each part. Each part contains their own constraints necessary to eliminate the variability that may be present across algorithms or systems. The design of the experiment will be discussed in detail in this section also outlining the constraints placed on entrants for each part.

\subsection{Participants}

The competition is open to all academic and industrial institutions. Upon registration, each participant is required to sign a database release agreement detailing the proper usage of data made available through the competition. Participants are then given a database access letter with a username and password to access the server to download the training data. In Table 1 are presented the participants names and the correspondent algorithms names as they're used in this paper. Four out of ten preferred to remain anonymous and the University of Naples Federico II submitted three different algorithms.

\subsection{Part 1: Algorithm Data Set}

The dataset for Part 1: Algorithms consists of images from four different devices; Biometrika, Crossmatch, Italdata and Swipe. There are 4000 or more images for each of these devices as detailed in Table 3 and 4 . The spoof materials used for this experiment were Body Double, latex, PlayDoh and wood glue for Crossmatch and Swipe and gelatine, latex, ecoflex (platinum-catalysed silicone), modasil and wood glue for Biometrika and Italdata. The images were divided into two equal datasets, training and testing. Details are described in Table 2 and Table 3 and 4. Live images came from 300 fingers from 50 subjects for Biometrika and Italdata datasets, 940 fingers representing 94 subjects for Crossmatch dataset, and 1000 fingers from 100 subjects for Swipe dataset. Spoof images come from approximately 225 fingers representing 45 people for the Crossmatch and Swipe Datasets and 100 fingers representing 15 subjects for the Biometrika and Italdata datasets.

The spoof images of two of the LivDet 2013 datasets (Crossmatch and Swipe) were collected using the cooperative method that was described earlier. The other two datasets (Biometrika and Italdata) were created using the non-cooperative method. This is the reason of the great

\begin{tabular}{|l|l|}
\hline Participants & $\begin{array}{l}\text { Algorithm } \\
\text { names }\end{array}$ \\
\hline Dermalog Identification Systems GmbH & Dermalog \\
\hline First anonymous participant & Anonym1 \\
\hline Universidad Autonoma de Madrid & ATVS \\
\hline Second anonymous participant & Anonym2 \\
\hline $\begin{array}{l}\text { University of Naples Federico II (first al- } \\
\text { gorithm) }\end{array}$ & UniNap1 \\
\hline $\begin{array}{l}\text { University of Naples Federico II (second } \\
\text { algorithm) }\end{array}$ & UniNap2 \\
\hline $\begin{array}{l}\text { University of Naples Federico II (third al- } \\
\text { gorithm) }\end{array}$ & UniNap3 \\
\hline Third anonymous participant & Anonym3 \\
\hline HangZhou JLW Technology Co Ltd & HZ-JLW \\
\hline Federal University of Pernambuco & Itautec \\
\hline Chinese Academy of Sciences & CAoS \\
\hline Fourth anonymous participant & Anonym4 \\
\hline
\end{tabular}

Table 1: Name of the participants and the submitted algorithms.

\begin{tabular}{|l|l|l|l|l|}
\hline $\begin{array}{l}\text { Data } \\
\text { set }\end{array}$ & Sensor & Model No. & $\begin{array}{l}\text { Resolu- } \\
\text { tion(dpi) }\end{array}$ & $\begin{array}{l}\text { Image } \\
\text { size }\end{array}$ \\
\hline$\# 1$ & Biometrika & FX2000 & 569 & $315 \times 372$ \\
\hline$\# 2$ & Italdata & ET10 & 500 & $640 \times 480$ \\
\hline$\# 3$ & Crossmatch & $\begin{array}{l}\text { L SCAN } \\
\text { GUARDIAN }\end{array}$ & 500 & $800 \times 750$ \\
\hline$\# 4$ & Swipe & & 96 & $208 \times 1500$ \\
\hline
\end{tabular}

Table 2: Device characteristics for Part 1 datasets

difference between the error rates: a fake created from a latent fingerprint (therefore without cooperation) may, in some cases, be less similar to the original one then one created with cooperation. After the competition is completed, the entire dataset will be made available to those who sign the proper data release agreement. Figure 1 below shows examples of images used in the experiments.

\subsection{Part 1: Algorithm Submission}

The algorithm submission for LivDet 2013 uses the same structure as LivDet 2009 and 2011. The four training sets of fake and live fingerprint images have been made available to 


\begin{tabular}{|l|l|l|l|}
\hline Data set & Sensor & $\begin{array}{l}\text { Live training } \\
\text { samples }\end{array}$ & $\begin{array}{l}\text { Live testing } \\
\text { samples }\end{array}$ \\
\hline$\# 1$ & Biometrika & $1000 / 200$ & $1000 / 100$ \\
\hline$\# 2$ & Italdata & $1000 / 200$ & $1000 / 100$ \\
\hline$\# 3$ & Crossmatch & $1250 / 500$ & $1250 / 440$ \\
\hline$\# 4$ & Swipe & $1250 / 500$ & $1250 / 500$ \\
\hline
\end{tabular}

Table 3: Training and test set characteristics for Part 1 datasets (live samples/number of fingers)

\begin{tabular}{|l|l|l|l|}
\hline Data set & Sensor & $\begin{array}{l}\text { fake training } \\
\text { samples }\end{array}$ & $\begin{array}{l}\text { fake testing } \\
\text { samples }\end{array}$ \\
\hline$\# 1$ & Biometrika & $1000 / 50$ & $1000 / 50$ \\
\hline$\# 2$ & Italdata & $1000 / 50$ & $1000 / 50$ \\
\hline$\# 3$ & Crossmatch & $1000 / 125$ & $1000 / 100$ \\
\hline$\# 4$ & Swipe & $1000 / 125$ & $1000 / 100$ \\
\hline
\end{tabular}

Table 4: Training and test set characteristics for Part 1 datasets (fake samples/number of fingers)

each participant, freely downloadable from the LivDet site after the participant registration. As stated for the other two LivDet editions each submitted algorithm must be a Win32 console application with the following list of parameters:

LIVENESS_XYZ.exe [ndataset] [inputfile] [outputfile] - [ndataset] is an identification number of the data set to analyze.

- [inputfile] is a text file with the list of images to analyze.

- [outputfile] is a text file with the output of each processed image, in the same order of [inputfile]. The result is a degree of liveness normalized in the range 0 and 100 (100 is the maximum degree of liveness, 0 means that the image is fake). In the case that the algorithm has not been able to process the image, the correspondent output must be -1000 (failure to enroll).

The submitted algorithms have been tested using each of the four testing sets. The threshold value for determining liveness was set at 50. This threshold is used to calculate error rates on live and fake fingerprints (FerrLive and FerrFake, see Section 3.5).

\subsection{Part 2: Systems Submission}

In Part 2: Systems, participants were asked to ship a fingerprint system which captures a fingerprint image as well as outputs a liveness detection score. Three spoof recipes and methods were made available to the participants upon their registration. These materials were Playdoh, Gelatin

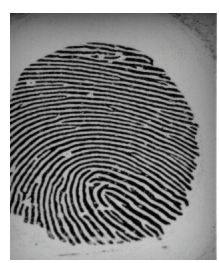

(a)

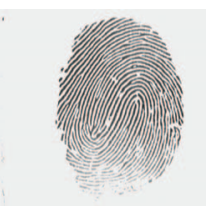

(d)

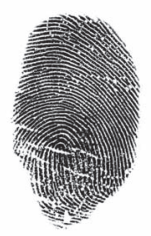

(g)

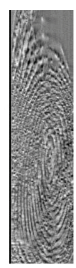

(j)

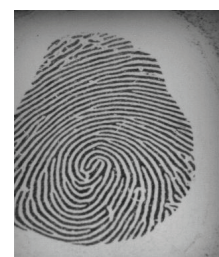

(b)

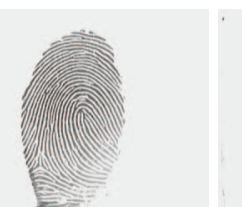

(e)

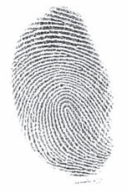

(h)

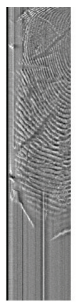

(k)

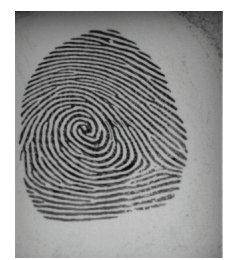

(c)

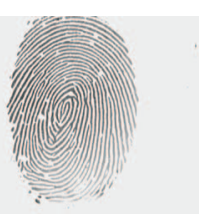

(f)
Figure 1: Examples of acquired fake fingerprints. Noncooperative: Biometrika (a, b, c); Italdata (d, e, f). Cooperative: Crossmatch (g, h, i); Swipe (j, k, l). Cast materials: gelatine (a, d); latex (b, e, h, k); wood glue (c, f, i, l); body double $(g, j)$.

and Ecoflex. In addition the systems were tested with two unspecified methods. These materials were Modasil and Latex. The requirements for installation are that the system will be run on either a Windows XP 32-bit or 64-Bit system, that the file will be an .exe or similar executable and that the system will use either a USB or Firewire connection. The system is required to output the collected image if the image is considered a live image and a liveness score normalized in the range of 0 and 100 (100 is the maximum degree of liveness, 0 spoof) for images detected as either live of fake. In the case that the algorithm has not been able to process the image it is considered an error for a live image and a success for a spoof image. Laboratory staff systemat- 
ically collected live data from participating subjects and attempted to spoof the system with casts made from the varying materials. Each submitted system was given 2000 test attempts. This corresponds to 1000 live attempts from 50 people ( 2 images each of all 10 fingers) were performed, as well as 1000 spoof attempts for the five different materials listed above. The spoof attempts were conducted with 2 images per finger from the 5 right hand fingers of 20 subjects for each spoof material. The same physical spoof fingers were placed on both scanners. A spoof image was collected on one scanner and then the next scanner alternating which scanner was first for a new spoof finger. In order to ensure quality of the spoof images, 15 spoof fingers were collected on a third party system to examine the quality of that batch of each spoof recipe.

\subsection{Performance Evaluation}

The parameters adopted for the performance evaluation will be the following:

\section{Evaluation per sensor/system:}

- Frej_n: Rate of failure to enroll for the sub-set $n$.

- Fcorrlive_n: Rate of correctly classified live fingerprints for sub-set $n$.

- Fcorrfake_n: Rate of correctly classified fake fingerprints for sub-set $n$.

- Ferrlive_ $n$ : Rate of misclassified live fingerprints for subset $n$.

- Ferrfake_n: Rate of misclassified fake fingerprints for subset $n$.

- ET: Average processing time per image.

\section{Overall evaluation:}

- Frej_n: Rate of failure to enroll.

- Fcorrlive_n: Rate of correctly classified live fingerprints.

- Fcorrfake_n: Rate of correctly classified fake fingerprints.

- Ferrlive_n: Rate of misclassified live fingerprints.

- Ferrfake n: Rate of misclassified fake fingerprints.

\section{Results and Discussion}

Eleven algorithms and two systems successfully completed the competition at the time of submission of this paper: Dermalog, ATVS, the University of Naples Federico II (three different algorithms), HZ-JLW, Itautec, CAoS and the three Anonymus for Part 1; Dermalog and Anonym4 for Part 2.

\subsection{Part 1: Algorithms}

The competition results are presented in the following tables, for the sake of space we only show the misclassified live fingerprints rate (ferrlive) in Table 5, the misclassified fake fingerprints rate (ferrfake) in Table 6 and the accuracy rate (the rate of images correctly classified) in Table 7.
Since, as stated earlier, the Biometrika and Italdata datasets were created using the non-cooperative method, the fakes were easier to detect therefore the error rates are much lower than those obtained with the Crossmatch and Swipe.

The live images collected with the Crossmatch sensor turned to be especially difficult to recognize for most of the algorithms. Tests of the Crossmatch dataset were conducted using our benchmark liveness detection algorithm. We received results similar to those presented by the other algorithms using the datasets as they were given to participants with error over $90 \%$. A second test was conducted switching one half of the subjects represented in the live training dataset with one half of the subjects represented in the live testing dataset. This reduced the error rate to $9 \%$. Further investigation is being conducted into why altering which subjects were in training and testing resulted in such a drastic lowering of the FerrLive.

The best performances were those of Dermalog with a 98.3\% accuracy for the Biometrika dataset, of Anonym2 with a $99.4 \%$ for the Italdata dataset, of the first Federico II algorithm with a $68.8 \%$ for the Crossmatch dataset and of Dermalog again with a $96.47 \%$ for the Swipe dataset. Finally, despite the great Dermalog and Anonym2 performances, the best average results were those of the first Federico II algorithm with a $86.63 \%$ of accuracy and with an average of $11.96 \%$ FerrLive and $14.62 \%$ FerrFake.

The Anonym1, Itautec and CAoS algorithms were not able to process the Swipe images and for this reason they did not compete for the final win despite the good performances of Anonym1 on the Biometrika and Italdata datasets. The failure to enroll rate was zero on all datasets for all algorithms except the Anonymous 1 one which has not been able to analyze one image from both the Biometrika and Italdata datasets and two images from the Crossmatch dataset.

\subsection{Part 2: Systems}

FerrLive and FerrFake for the two submitted systems can be found in Table 8. Dermalog performed at a FerrLive of $11.8 \%$ and a FerrFake of $0.6 \%$. Anonym 4 performed at a FerrLive of $1.4 \%$ and a FerrFake of $0.0 \%$. Both systems had low FerrFake rates. Anonym4 received a perfect score of $0.0 \%$ error, successfully determining every spoof finger presented as a spoof.

Figure 2 shows FerrFake and FerrLive by varying the acceptance threshold for the Dermalog system. We did not plot the analogous curve for Anonym4 system because it showed a FerrFake of $1.4 \%$ and FerrLive of $0.0 \%$ for all threshold values. Figure 3 shows the FerrFake rate for the Dermalog system for the unknown recipes and known recipes. Since Anonym4 had a $0.0 \%$ overall FerrFake, it did not require a plot. Dermalog performed considerably better against the known recipes than the unknown recipes. 


\begin{tabular}{|l|l|l|l|l|l|}
\hline & Biometr. & Italdata & Crossm. & Swipe & Average \\
\hline Dermalog & 3.30 & 0.50 & 99.84 & 3.82 & 26.86 \\
\hline Anonym1 & 1.50 & 0.50 & 86.96 & N.A. & N.A. \\
\hline ATVS & 4.60 & 0.00 & 90.40 & 0.00 & 23.75 \\
\hline Anonim2 & 2.30 & 0.20 & 98.40 & 2.52 & 25.85 \\
\hline UniNap1 & 3.00 & 2.10 & 31.28 & 11.45 & 11.96 \\
\hline UniNap2 & 1.80 & 5.00 & 55.20 & 33.22 & 23.80 \\
\hline UniNap3 & 1.80 & 2.10 & 55.20 & 11.45 & 17.64 \\
\hline Anonum3 & 3.30 & 1.00 & 95.52 & 2.69 & 25.63 \\
\hline HZ-JLW & 65.30 & 26.10 & 100.00 & 25.33 & 54.18 \\
\hline Itautec & 1.10 & 1.30 & 64.96 & N.A. & N.A. \\
\hline CAoS & 5.50 & 21.10 & 41.92 & N.A. & N.A. \\
\hline
\end{tabular}

Table 5: Rate of misclassified live fingerprints (ferrlive) for submitted algorithms

\begin{tabular}{|l|l|l|l|l|l|}
\hline & Biometr. $\mid$ Italdata & \multicolumn{2}{l}{ Crossm. } & Swipe & Average \\
\hline Dermalog & 0.10 & 1.10 & 0.00 & 3.20 & 1.10 \\
\hline Anonym1 & 2.40 & 1.70 & 2.40 & N.A. & N.A. \\
\hline ATVS & 5.50 & 100.00 & 10.30 & 100.00 & 53.95 \\
\hline Anonim2 & 1.30 & 1.00 & 0.30 & 9.60 & 3.05 \\
\hline UniNap1 & 6.40 & 4.90 & 31.10 & $16.10 \mid$ & 14.62 \\
\hline UniNap2 & 11.30 & 13.90 & 48.30 & $19.50 \mid$ & 23.25 \\
\hline UniNap3 & 11.30 & 4.90 & 48.30 & 16.10 & 20.15 \\
\hline Anonym3 & 8.10 & 4.60 & 0.10 & 8.20 & 5.25 \\
\hline HZ-JLW & 0.60 & 0.20 & 0.00 & 3.50 & 1.07 \\
\hline Itautec & 16.90 & 6.50 & 13.90 & N.A. & N.A. \\
\hline CAoS & 3.70 & 70.70 & 54.20 & N.A. & N.A. \\
\hline
\end{tabular}

Table 6: Rate of misclassified fake fingerprints (ferrfake) for submitted algorithms

The results from Dermalog continue to show the importance of learning different methods for creating spoof fingers as the knowledge of recipes helps to significantly lower the FerrFake rate. Figure 4 shows examples of accepted and rejected live images on Dermalog and Figure 5 shows examples of accepted and rejected spoof images on Derma$\log$. Anonym 4 did not record images in a format able to be viewed and thus images are not available.

\begin{tabular}{|c|c|c|c|c|c|}
\hline & & $\mathrm{a} \mid$ & Cro & Swipe & $A y$ \\
\hline $\mathrm{g}$ & & & & $96.47 \%$ & \\
\hline & & & & N.A. & \\
\hline & & $0 \%$ & 45. & & | \\
\hline & & 99 & & & 84 \\
\hline & & & & & 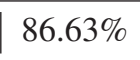 \\
\hline UniN & 03450 & $\mathrm{bl}$ & 4 & 6 & | \\
\hline & 93 & & 4 & 8 & 80. \\
\hline & & & & & $83.29 \%$ \\
\hline HZ-JLW & $67.05 \%$ & 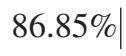 & 4 & $\%$ & 70 \\
\hline & $91.00 \%$ & $6 \mid$ & 5 & N.A. & \\
\hline CAoS & $95.40 \%$ & $54.10 \%$ & $52.62 \%$ & N.A. & $\mathrm{N}$ \\
\hline
\end{tabular}

Table 7: Rate of accuracy for submitted algorithms

\begin{tabular}{|l|l|l|l|l|}
\hline $\begin{array}{l}\text { Submitted } \\
\text { Systems }\end{array}$ & FerrFake & FerrLive & $\begin{array}{l}\text { FerrFake } \\
\text { Known }\end{array}$ & $\begin{array}{l}\text { FerrFake } \\
\text { Unknown }\end{array}$ \\
\hline Dermalog & $0.6 \%$ & $11.8 \%$ & $0.3 \%$ & $1.0 \%$ \\
\hline Anonym4 & $0.0 \%$ & $1.4 \%$ & $0.0 \%$ & $0.0 \%$ \\
\hline
\end{tabular}

Table 8: FerrLive and FerrFake for submitted systems

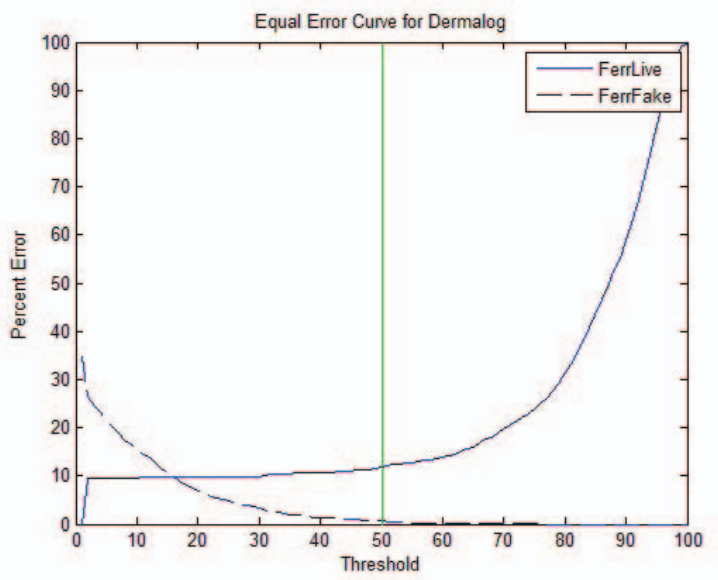

Figure 2: Equal Error Rate Curves for Dermalog.

Both systems had software built-in to auto-detect fingers and collect when a finger was found. All live fingers were able to be detected by both systems, however not all spoof fingers were detected. Dermalog had a 23\% Fake NonResponse Rate. The Anonym4 device had a 49\% Fake NonResponse Rate. It should be noted that a non-response is 


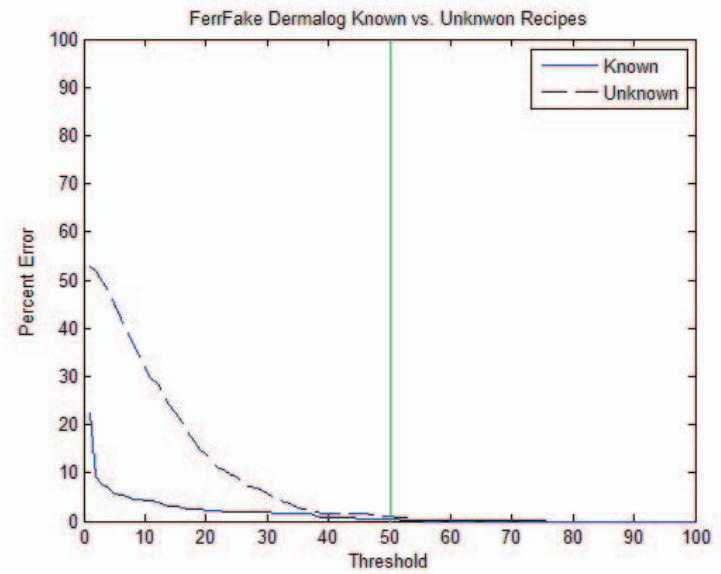

Figure 3: FerrFake for unknown vs. known recipes for Dermalog.

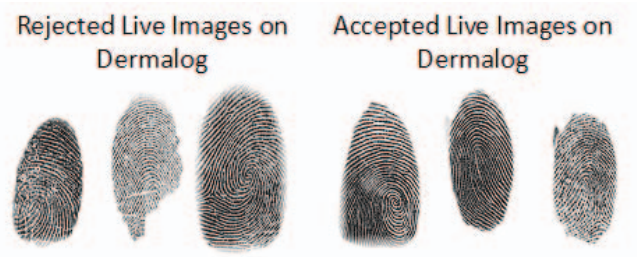

Figure 4: Accepted and rejected live images on Dermalog.

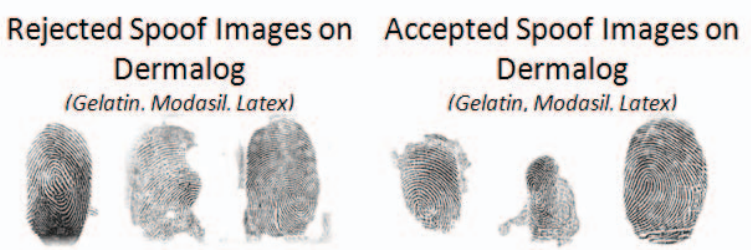

Figure 5: Accepted and rejected spoof images on Dermalog.

recorded as a successful detection of a spoof attempt. The high Fake Non-Response rate of Anonym4 can partially be attributed to the lack of moisture in some of the recipes. The system was unable to detect any Playdoh or Ecoflex images.

In addition to the testing reported here, a small subset of 50 images per material was conducted with the inclusion of a brushed on saline solution. The Anonym4 system was able to detect the previously undetected spoof fingers. However, even though the spoof was detected as a finger, none of the spoofs were detected as a live finger, i.e., there was no increase in FerrFake using the saline solution.

\section{Conclusions}

LivDet 2013, the third international public competition for software-based fingerprint liveness detection and the second public assessment of system-based fingerprint live- ness detection proved to be an important event in this field.

The number of participants, from both academic and industrial institutions, is growing with respect to previous editions. As a matter of fact entries were submitted from a total of ten participants.

Part 1 showed that, although fakes created with the non consensual method were detected with high accuracy, those created by the consensual method were much more difficult to recognize leading to a very high error rate. In Part 2, a high FerrLive value, and thus a high rate of live fingerprints rejected as fakes, could still be a problem when integrating fingerprint liveness detection methods on verification systems.

Since an effective liveness detection algorithms is a key component to minimize the vulnerability of fingerprint systems to spoof attacks, with this competition success we hope encouraging research from academic and industrial institutions on this challenging task.

\section{Acknowledgements}

This work was partly supported by the Tabula Rasa project, 7th FP of the European Union (EU), grant agreement number: 257289 and the Center for Identification Technology Research (CITeR).

\section{References}

[1] G.L. Marcialis, et al., First International Fingerprint Liveness Detection Competition, 14th Int. Conf. on Image Analysis and Processing (ICIAP 2009), Springer LNCS 5716, pp. 12-23.

[2] D. Yambay, et al., LivDet 2011 - Fingerprint Liveness Detection Competition 2011, 5th IAPR/IEEE Int. Conf. on Biometrics (ICB 2012), New Delhi (India), March, 29th, April, 1st, 2012.

[3] T. Matsumoto, et al., Impact of artificial gummy fingers on fingerprint systems, In Proceedings of SPIE, 4677, Optical Security and Counterfeit Deterence Techniques IV, Yokohama, Japan.

[4] P. Kallo, et al., Detector for Recognizing the Living Character of a Finger in a Fingerprint Recognizing Apparatus Patent US 6,175641, Jan. 16, 2001

[5] Schuckers SAC. Spoofing and Anti-Spoofing Measures. Information Security Technical Report, Vol 7. No. 4, pages 56-62, 2002.

[6] P. Coli, et al., Fingerprint silicon replicas: static and dynamic features for vitality detection using an optical capture device, International Journal of Image and Graphics, World Scientific, 8 (4) 495-512, 2008. 